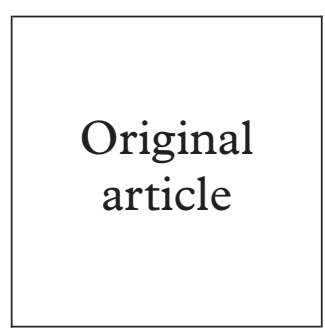

\title{
A social science perspective on screening for Chlamydia trachomatis
}

\author{
Barbara Duncan, Graham Hart
}

\begin{abstract}
A recent report from the chief medical officer's expert advisory group on Chlamydia trachomatis has recommended the setting up of two pilot projects to assess the feasibility of introducing a national chlamydia screening programme. In addition to screening all symptomatic individuals and all attenders at genitourinary medicine clinics, the report recommends opportunistic screening of sexually active young women and women at high risk of infection, who are attending either general practice or family planning clinics. The success of any new screening programme depends on a wide variety of factors, not least the acceptability of such screening to its target population. In recent years, social scientists have made significant contributions to the understanding of the psychological factors which facilitate or inhibit uptake of screening services. The aim of this report is to discuss briefly the contribution social scientists could make to the chlamydia screening programme in the United Kingdom. In particular, the possible effects of screening for a stigmatised condition such as a sexually transmitted infection are explored.
\end{abstract}

(Sex Transm Inf 1999;75:239-241)

Keywords: psychology; Chlamydia trachomatis; screening; sexually transmitted infections

\section{Introduction}

Chlamydia trachomatis is the most common, curable sexually transmitted infection (STI) in the United Kingdom. While treatment is simple and effective, detection is problematic owing to the largely subclinical nature of symptoms. Untreated or inadequately treated infection is a leading cause of pelvic inflammatory disease and subsequent reproductive morbidity. ${ }^{1}$ Screening programmes in other countries, such as the United States, have proved successful in reducing the prevalence of chlamydia in women, ${ }^{12}$ and a recent report by the chief medical officer has recommended the implementation of two pilot schemes in the United Kingdom. ${ }^{3}$ These will test the feasibility of opportunistically screening groups of women in community settings such as general practice and family planning clinics. (In this context, specific groups of asymptomatic women will be targeted; sexually active women under 25 , women undergoing terminations of pregnancy, and others at high risk of infection-for example, women between 25 and 35 with a new sexual partner or who have had two or more sexual partners in the previous year.)

The CMO's recommendations for screening in the United Kingdom include areas of research which will require collaboration between social scientists and health professionals. It is now well established that the uptake of screening services may be enhanced, and the possibility of adverse psychological effects reduced, if attention is paid to a number of identifiable cognitive and behavioural factors. ${ }^{4}$ However, the success of any new screening initiative depends on more than the utilisation of cognitive and behavioural factors which are generalisable from other screening contexts; for any new screening initiative, there will be areas of concern which are context specific. The aim of this paper is to introduce some of the impor- tant issues which arise from one such specific area of concern-the sexually transmissible nature of the infection.

\section{STIs and screening}

Participating in a screening programme is not simply a function of a cost-benefit analysis of objective health risk, but is "a personal experience embedded in a series of wider social contexts and processes." 5 There are specific psychological and social issues surrounding screening for a sexually transmitted infection (STI), particularly when that screening is sex specific. ${ }^{6}$ The proposed screening programme will take place against the prevailing sexual ideology — an ideology which stigmatises STIs, which mitigates against open discussion of sexual behaviour, and where the double standard of sexual behaviour (the acceptability of men's sexual activity versus the disease consequences of women's) is still current. The manner in which social and cultural attitudes towards sexual activity may affect chlamydia screening are unknown, but it is clear that they may have wide ranging ramifications for all aspects of the programme.

\section{Sexuality and cervical screening}

In recent years, research into the social impact of the national cervical screening programme has highlighted the link between perceptions of sexual activity and screening. While cervical screening differs from chlamydia screening in a number of important respects, two factors make comparison between the programmes interesting; both types of screening imply sexual activity and both are targeted at women. One significant issue which has emerged from this research is the impact of cervical abnormalities on women's perceptions of their own sexuality. It has been found, for example, that women tend to link cervical changes with 
perceptions of "deviant" sexual behaviour (for example, promiscuity) and experience feelings of contamination which have a negative impact on their self image and also on their sexual functioning. ${ }^{78}$ Women are also aware that it is only female sexual activity which is highlighted in the context of cervical changes; the contribution of men is largely ignored. From this perspective, cervical screening is not a neutral process, but one which provides a mechanism to reinforce existing inequalities. It has been argued that "...the potentially adverse consequences of sexual intercourse- $\mathrm{a}$ private event-can be surveyed and treated through screening services - a publicly based and funded system. ...it is women who transcend this private, public dichotomy and find their lives scrutinised in a manner alien to men...the focus is commonly upon women both as transmitters and contractors of relevant viruses...as both those whose cervixes are surveyed and whose sexual activity comes under surveillance" (p 16). ${ }^{9}$

We have suggested that cervical and chlamydia screening are similar in that both tests imply sexual activity. In fact, chlamydia screening does not simply imply sexual activity-screening for an STI by definition makes the connection between infection and sex explicit and unavoidable. We would argue that if women's perceptions of their own sexuality and sexual equality can be negatively affected by cervical screening (where the connection between sex and pathology might be seen as ambiguous) then the psychosocial impact for women of a sex based screening programme where sex and disease are inextricably linked is cause for concern and merits careful research. This research should encompass not only the possible psychological and social sequelae of screening, but the acceptability of such a programme both for recipients and for providers.

\section{The social acceptability of chlamydia screening}

An initial step in ensuring the success of any screening programme is recruiting participants into the programme. In the context of chlamydia screening, recruitment may present specific difficulties. The stigmatised nature of "aberrant" sexual behaviour which is implied by STIs imbues the act of screening with a meaning that displaces a cost-benefit health behaviour model of decision making about sexual health, where sexually active women are expected to attend for screening because they are "objectively" at risk. Concerns about reputation and self worth may become far more salient in the decision to attend than an objective measure of risk; for example, some women may reject invitations for chlamydia screening because they do not believe themselves to be "promiscuous" enough to be at risk, or they may fear being labelled as such if their test is positive.

\section{Screening in primary care}

It is clear from the above that recruiting women to take part in screening will be a sensitive task. Staff in primary care settings will need to develop skills which have, until now, been the prerogative of dedicated sexual health services such as genitourinary medicine clinics. At the very least, obtaining informed consent for screening will involve discussion about sexual behaviour. There is evidence that staff working in general practice are uncomfortable about discussing sexual health, as indeed are patients. $^{3}{ }^{10}$ In family planning clinics, where sexual health is perhaps a more salient topic than in general practice, a recent study found that less that $6 \%$ of clinic doctors had experience in genitourinary medicine. ${ }^{11}$ This may also have implications for their ability to discuss sexual health in the context of chlamydia screening.

Asking staff to integrate any new screening service into a workload which many already perceive to be overloaded ${ }^{12}$ will require careful assessment. In the case of chlamydia screening, successful implementation would make complex and difficult demands on the staff; regularly initiating the topic of screening for a stigmatised condition in a consultation which may be totally unrelated to sex will require a level of skill which, for some staff at least, may be problematic. Since recruitment is a necessary prerequisite of a successful screening programme, the educational needs of staff should be a priority.

\section{Conclusion}

One of the requirements of a successful needs assessment for any new screening programme is that context specific issues are identified and understood. The ultimate success of the chlamydia screening programme cannot be divorced from understanding and challenging the prevailing sexual ideology. If this is to be successful, researchers, policymakers, and health professionals must collaborate to change attitudes concerning one of society's last great taboos-sexually transmitted infections.

\section{Policy recommendations}

- Uptake of chlamydia screening services may be enhanced if the relevant psychological, social, and cultural factors associated with screening are identified and addressed

- A key area of research is the investigation of the way in which attitudes towards sexual activity may affect the uptake of screening by the target population

- Many women may not perceive themselves to be at risk of contracting chlamydia because of the stigma associated with a sexually transmitted infection. Addressing perceptions of risk (and stigma) will require public education

- Research suggests that staff in primary care settings may experience difficulties in discussing sexual health. If they are to recruit women into the screening programme, then their professional training needs must be addressed as a priority 
1 CDC. Chlamydia trachomatis genital infections-United States 1995. ҒAMA 1997;277:952-3.

2 Mertz KJ, Levine WC, Mosure DJ, et al. Trends in the prevalence of chlamydial infections. The impact of community-wide testing. Sex Transm Dis 1997;24:169-75.

3 Chief Medical Officer's Expert Advisory Group. Chlamydia trachomatis; summary and conclusions. London: Department of Health, 1998

4 Marteau TM. Towards an understanding of the psychological consequences of screening. In: Croyle RT, ed. Psychosocial effects of screening for disease prevention and detection. New York: Oxford University Press, 1995

5 Milburn K, MacAskill S. Cervical screening: continuing concerns in the 1990s. Health Educ F 1994;53:201-13.

6 Duncan B, Hart G. Sexuality and health: the hidden costs of screening for Chlamydia trachomatis. BMF 1999 318: 931-3.
7 Posner T, Vessey M. Prevention of cervical cancer: the patient's views. London: King Edward's Hospital Fund for London, 1988.

8 Fylan F. Screening for cervical cancer: a review of women's attitudes, knowledge and behaviour. $\mathrm{Br} \mathcal{F}$ Gen Pract 1998;48:1509-14

9 McKie L. Sex, sexuality and the cervical smear tests: dilemmas for the researcher and the researched. Medical Sociology News: Newsletter of the BSA 1993;18:15-22.

10 Rink E. Risk factors for urinary tract symptoms in women: beliefs among general practitioners and women and the effect on patient management Br F Gen Pract 1998;48:115-8.

1 Stect on patient management. Br F Gen Pract 1998;48:115-8. Sted genital-infection in women attending family-planning clinics

2 Chambers R, Belcher J. Work patterns of GPs before and after the introduction of the 1990 contract. Br f Fam Plan 1993;34:410-12.

\section{MSSVD Clinical Development Fund}

Call for submissions

The MSSVD invites submissions from members to support activities that advance the understanding and practice of genitourinary medicine which might not otherwise achieve funding. The maximum amount available is $£ 10000$. There is no lower limit. Applicants are encouraged to obtain matching funding from other sources, but this is not a precondition. Consideration will be given to supporting projects of the following type: Research; Clinical effectiveness; Visits to other centres; Sabbaticals. Priority will be given to members working outside major academic units and teaching centres.

The project must be supervised by a member of the MSSVD Council and progress reports made to the council every 6 months until completion.

Submissions to: Jessica Ribbing, MSSVD Secretariat, Royal Society of Medicine, 1 Wimpole Street, London W1M 8AE.

Further information: Dr Keith Radcliffe, Honorary Assistant Secretary, MSSVD (tel: 0121237 5719; fax: 0121237 5729; email: k.w.radcliffe@bham.ac.uk).

The deadline for receipt of submissions is Friday 20 August 1999. Applicants will be informed of the outcome in early November. 